\title{
Modelling of conjugate heat transfer in barium titanate plates heated by the air flow
}

\author{
Nikolay Kozyulin ${ }^{1,}$, Maxim Bobrov ${ }^{1}$, and Michael Hrebtov ${ }^{1}$ \\ ${ }^{1}$ Institute of Thermophysics of SB RAS, 630090 Ac. Lavrentiev ave. 1, Novosibirsk, Russia
}

\begin{abstract}
We present the results of simulation of conjugate heat transfer between the grid of barium titanate plates and the hot air flow. The air temperature undergoes rapid change and the thermal front propagation and heat exchange with the solid plates have been studied for several plate configurations. The results show that the air heat could be effectively absorbed by the plates during the time of thermal front propagation, making such configuration attractive for pyroelectric energy harvesting applications.
\end{abstract}

\section{Introduction}

The problem of conjugate heat transfer, when a solid is heated or cooled by the flow of gas or liquid has many practical applications, from environmental flows to engineering devices and power plants. At present, because of ecological needs and technical progress the development of environmental energy converters becomes more and more relevant. One way of utilizing thermal energy of the environment is by using pyroelectric effect $[1,2]$.

The pyroelectric effect is the change in electrical polarization of material with temperature. It can be used for generation of electric current and producing some electric power. For efficient power generation by this process the rapid change in temperature of the material is required. In practice this can be achieved by passing a volume of hot air through the grid of colder pyroelectric material [3].

In the present paper we concentrate on the numerical estimate of effective parameters of barium titanate $\left(\mathrm{BaTiO}_{3}\right)$ grid being heated by a hot air flow. The grid is constructed of flat plates of fixed longitudinal length placed on top of each other with some regular spacing between them. The grid spacing and plate thickness were varied in numerical simulations to find out the best parameters for the fastest and fullest heat absorption from the air.

\section{Computational details}

The single "cell" of the plate-grid was modelled. The computational domain contains two "half-plates" and the air channel in between (Fig 1). Each half-plate has symmetry conditions specified on the outer boundary and wall (i.e., no-slip) boundary conditions on

\footnotetext{
*Corresponding author: nikkozyulin@gmail.com
} 
the inner boundary where it contacts with the air flow. At the inner boundary the conjugate heat transfer conditions were set up, by balancing the heat flux between the solid and the fluid. At the inlet the constant air velocity profile was specified with the value of $15 \mathrm{~m} / \mathrm{s}$. This value was selected as practically achievable in experimental setups.

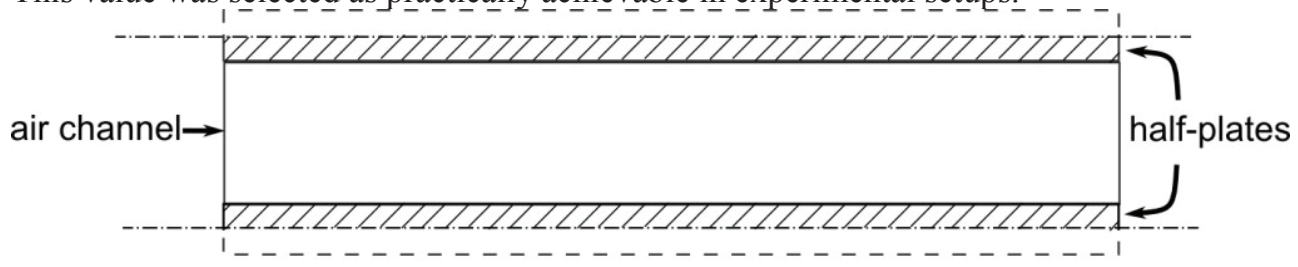

Fig. 1.The scheme of computational domain.

Air channel height (the plate-grid spacing) was varied between $0.1-2 \mathrm{~mm}$. The Reynolds numbers for this setup were between 75 - 1500. Taking into account the prescribed length of the channel $(2 \mathrm{~cm})$ for this small Reynolds numbers the three-dimensional turbulent effects in a spatially developing boundary layer are weak [4], thus we decided to perform a two dimensional simulation of the flow.

The simulation starts with the flow and the plates having the same temperature (273 $\mathrm{K})$, then at time $t_{0}$ the inlet air temperature rises fast by $100 \mathrm{~K}$ and the plates start to heat up. The inlet air temperature changes from $273 \mathrm{~K}$ to $373 \mathrm{~K}$ in $0.1 \mathrm{~ms}$, and the hot air front passes through the plates in $1.33 \mathrm{~ms}$.
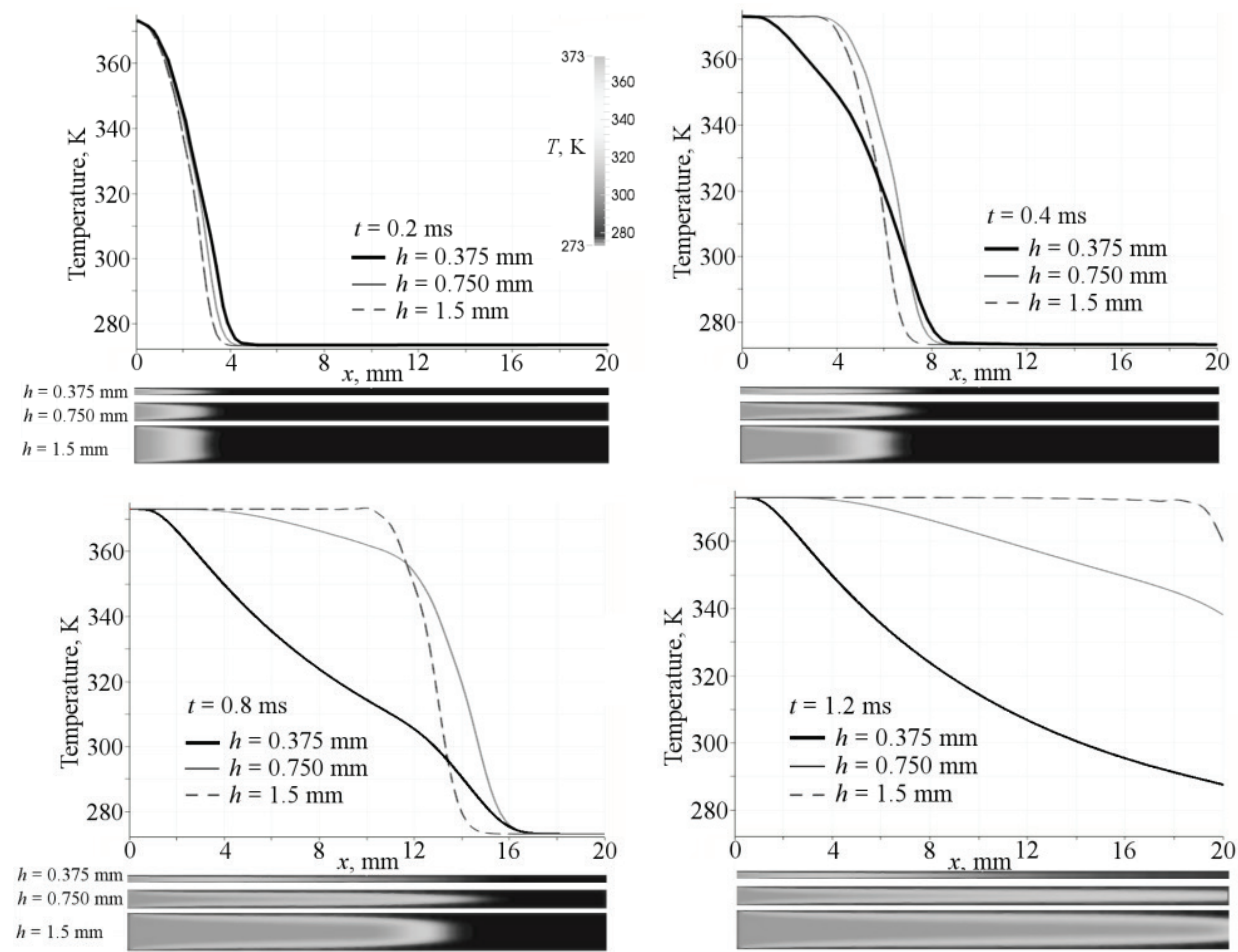

Fig. 2. The longitudinal temperature profiles through the central cross-section of the air channel for three channel heights at the different stages of passing of the hot temperature front. The bottom parts of the subfigures show the temperature distribution in the air channel. The plate thickness is 200 $\mathrm{nm}$. 
Three plate thickness values were tested: $20 \mu \mathrm{m}, 2 \mu \mathrm{m}$ and $200 \mathrm{~nm}$.

The domain has $220 \times 120$ computational points with clustering towards the inner walls in the air channel. In the solid part of the domain the grid spacing was uniform in both directions.

The $\mathrm{BaTiO}_{3}$ physical properties was taken from [5]. The computational code has finite-volume discretization with second order in space and time. No turbulence model was used. The buoyancy and compressibility effects were taken into account but were found to be negligible in the current simulations.

\section{Results and discussion}

\subsection{The effect of different plate spacing}

Figure 2 shows temperature field evolution in the air channel while the hot temperature front is proceeding through the channel. It can be seen that for the channel height $(h)$ of 1.5 $\mathrm{mm}$ the shape of the profile at the front is virtually not changing, reflecting that only a small fraction of heat is transferred to the solid plates in that case, thus rendering this spacing value inefficient. For smaller $h$ the shape of the profile starts to change with the hot front propagation progress. This is especially noticeable for $h=0.375 \mathrm{~mm}$, where the profile has two distinct parts, one (after the thermal front boundary) with evidently exponential behaviour, reflecting the constant heat loss to the plates, and the other with steeper slope at the thermal front boundary.
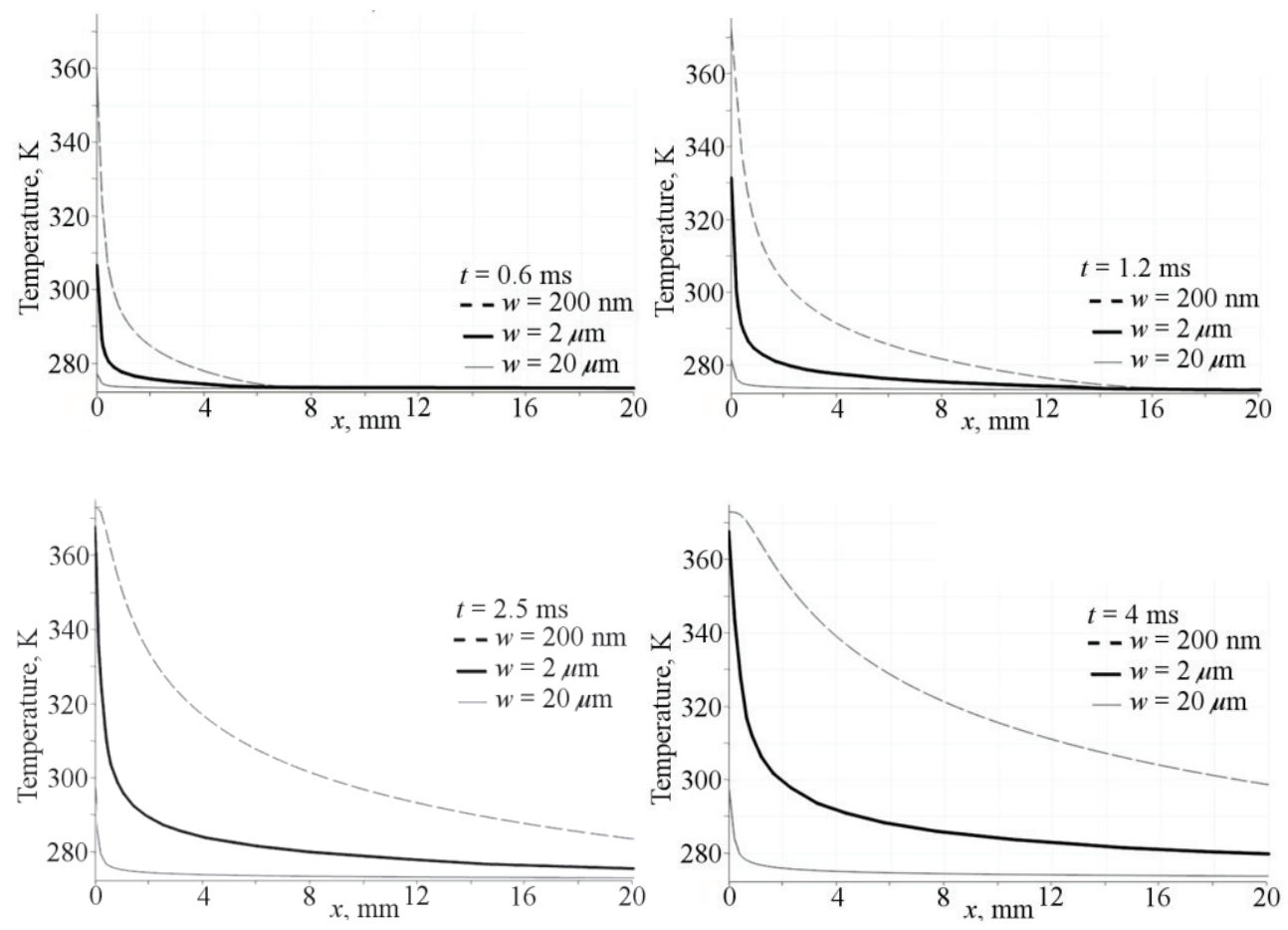

Fig. 3. The longitudinal temperature profiles through the central cross-section of the plate for three plate thickness values at the different stages of passing of the hot temperature front. The bottom part shows temperature distributions for times after the thermal front propagation. The air channel height is $0.75 \mathrm{~mm}$. 
After the front passes through the channel, the equilibrium temperature profiles are set up throughout the channel, varying slowly as the plates get more and more heated up. Another interesting feature is that smaller values of spacing lead to faster temperature propagation in the air. This is may be due to the mutual influence of the wall boundary layers, which is negligible for large $h(1.5 \mathrm{~mm})$, and becomes more pronounced for smaller $h(0.375 \mathrm{~mm})$.

As a result it can be seen that choosing the spacing of $0.375 \mathrm{~mm}$ leads to the transfer of major part of air thermal energy to the plates during the hot temperature front propagation which makes this setup promising for thermal energy harvesting.

\subsection{The effect of different plate thickness}

Figure 3 shows the temperature distribution in the plates during and after the heat front propagation. It can be seen that only for the thinnest plates (thickness $w=200 \mathrm{~nm}$ ) significant change in temperature is observed during the thermal front propagation time. The longitudinal distribution shows that the outlet parts of the plates are getting much less heated then the inlet parts for the prescribed channel length $(2 \mathrm{~cm})$.

In general, all of the tested plate configurations are not allowing the prescribed air flow to heat up the whole plate sufficiently during the time of thermal front propagation. Three or four times larger heating period is needed (Fig 3, bottom) to heat up even the thinnest $(200 \mathrm{~nm})$ of tested plates.

The thermal inertia of the plate leads to a very little sensitivity of the solid temperature field to the thermal front boundary position in the air.

\section{Conclusion}

The present study shows that the fast rise in the inlet air temperature may be effectively absorbed by the ferroelectric walls during the time of thermal front propagation if the plate spacing is small enough. In our case the plate spacing should be about $0.375 \mathrm{~mm}$.

The thermal response of barium titanate plates to the hot air flow with a steep thermal front was estimated numerically. The results show that for the fast temperature response in the plates which is needed for effective thermal power harvesting one is forced to use very thin plates (possibly coatings) of ferroelectric material in air heated setups. For reasonable air velocities (of the order of tens of $\mathrm{m} / \mathrm{s}$ ) the optimal plate sizes might be calculed. Our results show that the chosen plate length of $2 \mathrm{~cm}$ is too high, and should be shortened that will lead to a faster growth of the plate temperature and less pressure drop in the air channel.

The optimal parameters for the specified air velocity of $15 \mathrm{~m} / \mathrm{s}$ and the temperature difference of $100 \mathrm{~K}$ could be the following: the plate length of about $1 \mathrm{~cm}$, the plates spacing of $0.375 \mathrm{~mm}$ and the plate thickness of $200 \mathrm{~nm}$ or less.

This work was supported by Russian Science Foundation (grant №16-19-00119).

\section{References}

1. S.R. Etesami, J.Berakdar, Appl. Phys. Lett., 108 (2016)

2. M. Sharma, A. Chauhan, R. Vaish, V. S. Chauhan, Solar Energy, 115 (2015)

3. D. Vanderpool, J. H. Yoon, L. Pilon, Int. J. Heat Mass Tran, 51 (2008)

4. M. Lesieur, Turbulence in fluids (Springer Science \& Business Media, (2012)

5. Y. He, Thermochimica acta, 419 (2004) 